\title{
«Il cor si finge un ghiaccio e in fuoco giace»: amore e gelosia nella produzione lirica di Margherita Costa
}

\author{
[...] che non è in somma amor, se non insania, \\ a giudizio de' savi universale. \\ L. Ariosto, Orlando Furioso, XXIV, ${ }^{1}$
}

\begin{abstract}
Il presente contributo propone una lettura macrotestuale delle prime raccolte liriche di Margherita Costa (1600 ca.-1657): La Chitarra e Il Violino (1638). I due canzonieri barocchi presentano similarità strutturali (false indicazioni editoriali, polimetria, elementi paratestuali simmetrici) e tematiche (liriche amorose, encomiastico-celebrative, burlesche) e, in entrambi, è attribuito un ruolo predominante alla materia amorosa. L'io lirico, rappresentato nella quasi totalità da una bella donna, è vittima della potenza distruttiva dell'amore, tormentato da una spropositata passione amorosa che non riesce né ad arginare né a soddisfare. Dal caleidoscopio elegiaco offerto dai due volumi, l'attenzione sarà rivolta verso due tematiche: la malattia d'amore e la gelosia, declinate dalla poetessa emulando la tradizione lirica precedente. In particolare, si riconoscono profondi debiti verso Giovan Battista Marino, di cui vengono riproposte strutture metriche, sintattiche e retoriche e da cui sono tratti spunti tematici. Rispetto ai modelli, tuttavia, la virtuosa scrive versi dal marcato carattere drammatico, debitori della sua carriera di cantante e, giocando con le aspettative gender del lettore, procede in un'inversione dei ruoli del mittente e del destinatario per donare, in un contesto misogino, una voce femminile al genere del lamento amoroso.
\end{abstract}

The e-journal «altrelettere» is hosted at the URL: http://www.altrelettere.uzh.ch, in accordance with the Open Access Policy of the University of Zurich. Please cite this article as follows: M. DI MARO, «Il cor si finge un ghiaccio e in fuoco giace»: amore e gelosia nella produzione lirica di Margherita Costa, in «altrelettere», (2021), pp. 43-74, DOI: 10.5903/al_uzh-53.

(C) This article is licensed under a Creative Commons Attribution 2.5. Switzerland (CC BY-NC-ND 2.5). Please read the license terms on the website: http://creativecommons.org/licenses/by-nc-nd/2.5/ch/deed.en 
Un palcoscenico di carta: La Chitarra e Il Violino

Tra i numerosi temi presenti nella vasta produzione di Margherita Costa è indubbiamente opportuno riconoscere un ruolo predominante alla tematica amorosa. ${ }^{1}$ La virtuosa propone una variazione testuale, una «trasformazione con vari artifici ritmici, armonici, timbrici di un elemento tematico di base» (GDLI, 675). Costa, infatti, esacerba, quasi fino alla noia, il racconto di un'universale vicenda amorosa, in cui un io lirico ferito grida a gran voce la sua sofferenza e tesse un feroce vituperio nei confronti della persona amata. Seguendo le forme musicali coeve costruite proprio sulla tecnica della variazione, la poetessa propone un ostinato armonico della dinamica amorosa sui toni dello sdegno e della gelosia.

In questa sede prendo in esame i primi due canzonieri pubblicati dalla Costa, La Chitarra e Il Violino, i quali, presentando vicinanze tematiche e strutturali, possono essere oggetto di una lettura macrotestuale. ${ }^{2}$ Le due raccolte, infatti, sono pubblicate con le stesse false indicazioni editoriali,3 nello stesso anno (1638) a distanza di pochissimi mesi - aprile La Chitarra e giugno Il Violino e sono dedicate entrambe al Gran Duca di Toscana Ferdinando II (Stumpo 1996). Entrambe si aprono con autodichiarazioni, ironiche e provocatorie, sullo scarso valore dei versi pubblicati ed entrambe sono precedute da sonetti d'encomio (come quelli di Alessandro Adimari e Ottavio Tronsarelli) che dimostrano il consenso e il prestigio di cui la virtuosa godeva sia nella corte fiorentina che in quella romana (Di Maro 2020). Agli elementi paratestuali vanno aggiunti elementi interni: le sezioni sono scandite metricamente, a discapito dell'ormai diffusa suddivisione tematica proposta dalle Rime di Torquato Tasso e consolidata da La lira di Giovan Battista Marino (Martini 1984 e 2002; Tomasi 2015) e blocchi di liriche d'amore si intrecciano con componimenti encomiastico-celebrativi e motivi burleschi. Se si osservano le forme poetiche presenti in entrambe le raccolte, sembra quasi che con la pubblicazione de Il Violino Costa tenti di riempire un ‘vuoto metrico' lasciato nella precedente. Infatti, come si evince dalla Tabella 1, la netta superiorità numerica delle ottave 4 e dei sonetti nella prima lascia poco spazio a idilli e canzonette, che invece diventano predominanti nella seconda. 


\begin{tabular}{|l|l|l|}
\hline $\begin{array}{l}\text { Forma } \\
\text { metrica }\end{array}$ & La Chitarra & Il Violino \\
\hline Ottave & 95 & 1 \\
\hline $\begin{array}{l}\text { Capitolo in } \\
\text { terza rima }\end{array}$ & 2 & $/$ \\
\hline Idilli & 10 & 17 \\
\hline Sonetti & 95 & $/$ \\
\hline Canzonette & 23 & 25 \\
\hline
\end{tabular}

Tabella 1

Nel gioco di rimandi creato dall'autrice - presente anche ne Lo Stipo (1639) la similarità strutturale delle due raccolte trova riscontro in quella tematica: entrambe contengono descrizioni delle varie forme del sentimento d'amore, spesso condite con una vena umoristica e satirica, che vengono usate come strumenti per costruire una 'stanza tutta per sé' - per usare la celebre formula di Virginia Woolf - nella tradizione lirica peninsulare. Lo scenario è sempre lo stesso: l’io lirico, rappresentato nella quasi totalità da una bella donna, inveisce contro un amante crudele, che non si cura del suo affetto e del suo tormento e spesso rivolge i suoi favori ad altre donne; si lamenta per la partenza dell'amante o per la sua assenza; è vittima della potenza distruttiva dell'amore e/o si presenta spesso come un'amante «di sdegno» 5 e dunque, irata, indignata, afflitta e tormentata da una spropositata passione che non riesce né ad arginare né a soddisfare. Intrappolato in una coazione a ripetere, l'io lirico ha difficoltà a svincolarsi dai lacci d'amore e riconosce nel lamento elegiaco l'unico sollievo a tale pena. Le liriche, inoltre, presentano un marcato carattere drammatico, debitore della carriera di attrice e cantante della loro autrice. Si tratta, ovviamente, di una teatralità implicita perché Costa ricorre a strumenti derivati dalla pratica teatrale che rendono il discorso, seppur elegiaco, più dinamico e provano a riprodurre lo scambio di battute a due voci o le modalità del soliloquio. Numerosissime, infatti, sono le apostrofi indirizzate al destinatario dei lamenti e delle invettive (spesso segnalate tipograficamente con l'uso di parentesi), ma anche ai 'fedeli d'amore' che condividono o bramano di condividere tali pene; altrettanto numerose sono le proposizioni interrogative ed esclamative, in una complessiva enfatizzazione della funzione fatica.

Va, però, riconosciuto sin da subito che Costa non apporta grandi novità agli elementi caratterizzanti il topos della sofferenza d'amore e, ad una prima lettura complessiva delle sue raccolte, queste liriche sono messe in ombra da altre 
meno tradizionali e costruite intorno a temi quali la maternità (spesso sofferta), la condizione femminile, la violenza e la rivendicazione con toni satirici di uno spazio muliebre autonomo nella società misogina coeva (Di Maro 2019; De Liso 2020). Tuttavia, la presenza di un messaggio altamente codificato non impedisce all'autrice di muoversi liberamente: servendosi di questo, infatti, propone un racconto delle pene d'amore da una prospettiva diversa, appropriandosi di temi e argomenti tradizionalmente riconosciuti come prerogativa maschile. ${ }^{6}$ L'analisi attenta di queste liriche, poi, permette di individuare procedimenti drammatici precisi, che si ripetono in più punti delle raccolte, di inserire a tutto campo la sua autrice nel milieu di riferimento, di rintracciare i punti di contatto tra produzione lirica e attività da virtuosa e soprattutto di individuare i numerosi debiti contratti con i suoi modelli. Pertanto, suddividerò l'analisi tematica in due parti, concentrandomi rispettivamente sulla malattia d'amore e sulla gelosia e presentando, poi, i punti di contatto con Giovan Battista Marino.

«Si sta male d'amore, ma non si more! / È sciocca malattia, mal nata pena!»: signa e cura dell'amor hereos

La malattia d'amore (CIAVOLELLA 1976; PERI 1996; TONELLI 2015) è una formula ambivalente nella storia della medicina: essa, infatti, può comprendere sia le malattie veneree (sifilide o gonorrea), sia le affezioni della mente, che possono provocare gravi disturbi fisiologici (palpitazioni, pallore, fastidi uditivi e visivi), comportamentali (insonnia, inappetenza, ciclotimia) o mentali (depressione e tendenze suicide). Questi sono i sintomi dell'Amor hereos, secondo un'etichetta utilizzata, specialmente dai medici tardorinascimentali, per designare un'esperienza patologica ben nota a quegli innamorati che assolutizzano il proprio oggetto d'amore (PomA 2007).

Arnaldo da Villanova ricopre un ruolo fondamentale nella cristallizzazione dell'Amor hereos nella tradizione lirica italiana. Nel suo trattato, De amore heroico, ${ }^{7}$ il medico di Montpellier chiarisce la definizione, descrive le cause (causa), i sintomi (signa) e la terapia (cura) del mal d'amore: 
Morbus etenim est innaturalis dispositio, seu contra naturam membri existit nocumentum, aut quod ex dicta mala dispositione sequitur ad actione virtutis operantis in organo, si contra naturam dispositio convenienti nomine morbi accidents appellatur. Amoris igitur, cum non sit male dispositi membri, sed potius nociva actio, seu mala virtutis operanti organo, quae causerunt ex eiudem dispositione contraria [...]. Dico ergo [...] quod amor talis, videlicet qui dicitur hereos, est vehemens et assidua cogitatio supra rem desideratam cum confidentia obtinendi delectabile apprehensum ex ea» (Villanova 1585, 1524-25; corsivo mio)

Hereos non è una malattia (morbus) ma un sintomo (accidens), un'affezione che altera l'equilibrio degli umori (in particolare della bile gialla) ed è provocata da una riflessione intensa e costante sull'oggetto desiderato, nutrita dalla speranza di trarne godimento. L'immagine mentale ossessiva si appropria della cavità mediana del cervello (sede di formazione dei giudizi) e da qui domina tutto il resto del corpo. Il cervello, poi, è colpito anche dai vapori del cuore, prodotti dall'eccesso di passione, i quali seccano e raffreddano le sue cavità, originando delle qualità fisiologiche - il freddo e il secco - che contribuiscono a fissare le immagini mentali e che dispongono quindi inevitabilmente alla sofferenza. Questo tipo di affezione, però, colpisce maggiormente le persone attive (promptiores), mentre gli stessi sintomi e le stesse modalità potranno generare un amore malinconico negli individui di indole, appunto, malinconica (remissi).

Il processo diagnostico deve, però, essere tempestivo perché hereos può presto degenerare in forme pericolose, come la malinconia, la follia o addirittura la morte. La descrizione delle cause e dei sintomi della malattia è seguita, come in tutti i trattati medici, dall'anamnesi e dalla prescrizione della cura, nel caso specifico a contrarïs. Quando sia impossibile concretizzare un rapporto sessuale - l'unica soluzione efficace per tale affezione -, Villanova consiglia cure 'universali' come ascoltare musica, fare una passeggiata o organizzare un viaggio e, soprattutto, propone una soluzione molto semplice:

[...] forma itaque recta curationis habetur, si contrarium haiusmodi furie distractium diligentia procupetur inducit. Cum igit hec furrua, fui causa formalis sit intensa cogitatio super delectabile, hoc cum confidentia obtinendi, erit illis directio e correctium oppositum, non in hoc delectabile cogitare, nec sperare illo modo eius obtenunt. Hec vero perficient competenter, quaecunqui per 
representationem suarum formarum in virtute phantastica distrahunt in diversum a predicta cogitatione in toto, vel in parte aliqua parte saltem, veluti forma rerum, ducentium rem desideratam in odiu sicut rei turditudines oculo monstrare, vel enarrare sermonibus. (ibidem, 1530; corsivo mio)

Se la causa dell'hereos è un intenso desiderio verso qualcosa che non si può avere, l'unica terapia possibile è sviluppare verso di esso ripugnanza o operarne la denigrazione: il medico consiglia di indurre la facoltà immaginativa a funzionare alla rovescia e 'costringerla' ad immaginare qualità e comportamenti odiosi e perniciosi dell'oggetto amato.

Nelle liriche amorose di Costa sono presenti i signa e la cura ab contrarïs all'amor hereos. Tuttavia, è necessario precisare sin da subito che non è necessario supporre che l'autrice abbia letto il trattato medico, dato che questo le si presenta come fonte secondaria: a partire dal XIII secolo, infatti, la materia lirica sulle pene d'amore ricava da Villanova la descrizione dei sintomi di una vera malattia, le sue nefaste conseguenze e la cura. La romana, del resto, ha letto di amanti preda del mal d'amore, canta in scena i dolori e le angosce provocate da questa malattia e conosce bene gli 'affetti' declinati da Tasso e da Marino (suoi principali modelli di riferimento) nelle loro liriche d'amore. Ł̀ dalla secolare tradizione lirica, dunque, che l'autrice ricava i materiali per il suo racconto, in cui una bella donna, io poetante nella quasi totalità dei componimenti, ${ }^{8}$ è «preda d'ogni amoroso affanno», prova «pena e cordoglio» perché «avvinto in duri lacci il [suo] Core, / forsennato d'amor, arde amore» (CosTA 1638a, 80-82). La sintomatologia dell'Amor hereos è, infatti, presente in diversi suoi componimenti: i rimandi interni, costruiti sia a livello lessicale e retorico che tematico, permettono di leggere questi testi in modo unitario. Riporto, dunque, una casistica puramente esemplificativa.

Il pallore, gli occhi umidi e il tremore tratteggiano la fisiognomica della donna innamorata (signa):

pallore Ognun mi dice ch'io son distrutta e ch'infelice divengo brutta [...] 
il mio pallore
il mio sembiante
è sol d'amore
fido e costante;
porto pallido il volto e 'l petto acceso,
il piè disciolto, il cor legato e preso.
(idill. 14, vv. 1-12: CosTA 1638b, 151-52);

occhi umidi / Mi vedi abbandonata in queste piume, pianto e quasi il spirto mio vedi spirante; mi vedi esangue e l'uno e l'altro lume, mi vedi ogn'hor mancar'a te d'avante; mi vedi scaturir d'agl'occhi un fiume, mi vedi nel morir a te costante; e tu per secondare l'empio desire, cerchi con doppio duol farmi morire. (ott. 66, 4: CosTA 1638a, 255);

tremore
Stupida e fissa come in certa sabbia mi duolgo ogn'hor dei miei desir mal nati; col cor tremante e con immote labbia, languidi gl'occhi al Ciel tengo levati. Sdegnata accuso il gran motor che m'habbia tutti inclinati a mio sol danno i fati, e immota e come attonita sto alquanto, poi sciolgo al duol la lingua e gl'occhi al pianto. (ott. 62, 12: ibidem, 245).

Disturbi comportamentali e mentali, quali l'insonnia, la solitudine, il desiderio di morte, la sofferenza per la lontananza dall'amato, lo sdegno, scandiscono il canto della voce protagonista:

Ch'io dorma (iniquo) ahi come sia mai vero!
Se tu togli al mio Cor la pace antica,
tu dagl'occhi mi squarci il velo nero,
che già godei con tanta fede amica;
tu degl'affetti miei ti mostri altero,
onde la fama contro me ridica,
ch'io degl'ardori miei son disleale
e fuggo il bene per seguire il male.
(ott. 75,3: ibidem, 305);


Solitudine

desiderio

di morte

sofferenza

per la

lontananza

sdegno
Qui fuor d'ogni piacer, fuor d'ogni gioco

in solitario albergo i giorni e l'hore

traggo miseramente in'aureo loco

in dura servitù, sciolta d'amore;

il volto resta esangue à poco à poco,

il spirto già vien meno, il brio si more

e gl'occhi, che fur scorta à mille amanti, adombrati si fan, scorta di pianti.

(ott. 24, 2: ibidem, 110);

Morte, morte pietosa,

trafiggi questo petto,

trafiggi questo seno,

tronca, tronca ti prego

il filo del mio stame

e con la morte almeno

non mi negar (ohimè) pietosa aita!

(idill. 9, vv. 116-122: CosTA 1638b, 67);

Come vuoi ch'io sia lieta a te lontano?

Come posso io gioir senza vederti?

Come vuoi ch'io sopporti un duol si strano?

Come goder ti posso e non goderti?

Che posso mai sperar se spero invano?

Come sei mio, se mio non posso haverti?

Ah, ch'i contenti miei non son contenti, ma perversa cagion dei miei tormenti.

(ott. 28, 2: COSTA 1638a, 124);

Mi pagherai col sangue e con la vita il tradimento, iniquo; il Cielo, il Cielo ti punirà se m'hai così schernita.

Il ciel ti toglierà dagl'occhi il velo, in darno chiederai pietosa aita;

s'hor in darno io mi duolgo e mi querelo, mi pagherai fra l'ombre quel che devi e al foco andrai, se già nel foco ardevi.

(ott. 72, 37: ibid., 298).

Nel racconto che si svolge tra le pagine dei canzonieri, la malattia d'amore, però, non degenera nella sua forma più grave, la malinconia, anche perché la sua vittima rientra nella categoria dei promptiores, degli attivi, e l'io lirico femminile non si lascia sopraffare dall'affezione. Infatti, pentita della sua 
benevolenza verso un indegno amante, riconosce il suo errore di giudizio, si interroga a più riprese sull'utilità di un amore non ricambiato, si chiede perché serrare il proprio cuore in una morsa di sentimenti che si riveleranno, inevitabilmente, fonte di dolore. ${ }^{9}$ Per questo motivo, comincia a costruire come suggerito dai medici - il suo pensiero ab contrariis: reagisce alla malattia elencando a sé stessa i difetti e le manchevolezze dell'amato, maledicendolo, augurandogli la morte e promettendogli le stesse sofferenze:

Terra non trovi che l'accoglia in seno, cibo non sia che lo nutrisca o curi, sol sia suo cibo un'urna di veleno. Regimento li siano i suoi spergiuri, il sol per lui non sia mai più sereno; lo spaventino ogn'or portenti oscuri e il ciel gli nieghi pace e il Mondo aita; Morte porti ogn'or morte alla sua vita. (ott. 48, 9: ibidem, 204);

Sì, sì (perfido) sì si ch’io ti voglio soggiogar sotto me, sotto i miei lacci, sì, menzognero sì, ch'il duro scoglio del petto tuo per me sarà che sghiacci; sì (dispietato) sì ch'il tuo cordoglio sarà che per me sol l'alma si stracci e accinto ai lacci miei sì che vivrai finché vivo del Sol tu miri i rai. (ott. 66, 31: ibidem, 264).

Rientrano tra le strategie curative $a b$ contrariis anche i feroci attacchi che la donna muove verso il suo uomo mobile, che non si cura dell'amore e si mostra sempre ingrato, infedele e incostante. Insomma, alla controparte maschile vengono attribuite quelle caratteristiche che topicamente erano conferite solo alla donna:

ingratitudine Che vuoi da me? Che più ti posso dare se con il cor ogni mio ben ti diedi?

L'alma ti soggiogai che più bramare 


ti resta (iniquo) che 'l mio ardor non credi?
Ahi, che solo è tua gioia il mio penare
se più sbrami il petto al tuo rigore?
Vuoi dar la morte a chi ti diede il Core?
(ott. 47, 7: ibidem, 199).
[...] A me tormenta Amor per mia follia,
per troppo osservar fede a un infedele,
per un ch'ogn'hor mi porge Tosco e Fiele
e solo gode della pena mia.
Per un senza cervello, un senza tratto,
un sciocco, un scemunito, un traditore,
un ch'ad ogn'hora più s'accosta al matto. [...]
(son. 89: ibidem, 489).
Non più niego il mio mal, son fatta Ancella
del più superbo e dispietato Amante
che sostenga qua giù perversa stella
di cor mentito e d'animo incostante.
Ha finto riso e spergiura favella
volubilmente e in affermate piante,
e più del Re dell'ombre ha in seno orrore
di se rubello e sprezzator d'Amore.
(ott. 49, 4: ibidem, 206).

Ma le invettive sono rivolte anche al suo cuore ostinato che, aprendosi all'amante sbagliato, non riesce ad arrestare il suo funzionamento, non riconosce il suo «infelice stato» (ott. 56, 2: ibidem, 225) e «tanto cieco [è] nel [s]uo desire / che per folle gioir corr[e] a morire» (ott. 93,3: ibidem, 375). L'io lirico, dunque, si affranca dal dolore grazie al canto e trova sollievo nelle invettive rivolte verso chi causa le sue tribolazioni, i cui sintomi sono riconosciuti, nei rari momenti di lucidità, come innocui accidenti di una malattia sciocca e non insidiosa:

E che forse credevi ch'al mio Core non sapessi discior l'aspra catena? Si sta male d'amore, ma non si more! È sciocca malattia, mal nata pena! Tu già sai che lo sdegno vince amore e ogn'un sdegna di solcar la rena, 
donna già nacqui ove superbia regna

l'esser sola in amore Donna disdegna.

(ott. 84, 10: ibidem, 340; corsivo mio).

Una malattia di carta, insomma, che resta pericolosa solo nella finzione letteraria.

$\mathrm{O}$ crudel mostro, o peste de' mortali, / che fai li giorni miei sì oscuri e tristi? ${ }^{10}{ }$ : il cor ghiacciato dell'amante geloso

Margherita Costa, dunque, accosta la malattia e la sofferenza d'amore alla figura della bella donna, ribaltando i ruoli di amato/amante, sovvertendo la topica tradizionale e facendone provare i sintomi a colei che di norma ne era la causa nella tradizione medico-letteraria. Nel suo corpus lirico di tema amoroso anche la gelosia occupa un posto di rilievo. Del resto, tra Cinque e Seicento questa passione è oggetto di numerose descrizioni (Cherchi 1992, Gundersheimer 1993, Milburn 2002): liriche, discorsi, trattati, dialoghi e pièce teatrali sono dedicati a quell'affezione dell'animo che Marino definisce «Cruda ministra di cordogli e pene, / propizia al male et aversaria al bene» (XII, 2, vv. 7-8: Marino 2013, 1182). Inoltre, dopo la stagione del petrarchismo, la gelosia è protagonista di una graduale femminilizzazione, specialmente in ambito teatrale11 (Micali 2007), mentre con Tasso e Marino diventa una parte indiscussa del repertorio lirico, riconosciuta come una conseguenza inevitabile dell'amore, necessaria per intensificarlo e renderlo più duraturo.12 Determinanti in questo processo sono le numerose lezioni accademiche13 sul tema e, tra queste, il Discorso sulla gelosia14 di Torquato Tasso, che gioca un ruolo decisivo nella fortuna, diffusione e rappresentazione del topos nel XVII secolo (Prandi 2014; D’Amico 2015). Qui il sorrentino delinea una 'storia letteraria' della gelosia e, soprattutto, attribuisce ad essa il valore di affetto, e dunque la riconosce come un'affezione provocata da una causa esterna e subita passivamente dall'animo.15 Il Discorso, infatti, è una sintesi delle teorie sulla gelosia costruita su un'equilibrata commistione tra tradizione filosofica antica (Aristotele e Platone), tradizione medievale (Petrarca e Boccaccio) e fonti contemporanee (Ariosto, Della Casa, Tansillo). L'argomentazione tassiana si divide in quattro 
parti: in primo luogo, il poeta cerca di definire l'origine e la natura della gelosia, giungendo alla conclusione che la sua sostanza è la paura. Nella seconda parte, ne specifica la natura, distinguendola da invidia, disperazione ed emulazione; nella terza, il poeta - che recupera le riflessioni di Lorenzo de' Medici (Selve) e Sperone Speroni (Dialogo d'amore) - si chiede se amore e gelosia siano o meno inseparabili evocando le origini mitiche di queste passioni. La quarta, e ultima parte, contiene molti spunti interessanti per comprendere le sue trasposizioni simboliche e riprende l'immaginario allegorico fornito da Boccaccio nel Filocolo (III, 24):

Dice egli [Boccaccio], che l'albergo della gelosia è situato in una dell'altissime rocche dell'Apennino, in una oscurissima grotta circondata da ogni intorno di neve, combattuta dal vento, a la guardia della quale stanno sempre vigilanti due grandissimi cani; ch'ella va vestita a bruno; che di sesso è femina, di età vecchia, di color livida, di disposizione del corpo magra; che si scaldava ad un foco dove rilucevano due quasi estinti tizzi; che dormiva sul liminal dell'uscio fra' duo suoi cani. Da questa descrizione si possono facilmente comprendere molte sue proprietà (TASSO 1875, 184).

Nel paragrafo successivo, l'autore spiega il passo boccacciano - che si ispira alla descrizione della casa dell'Invidia nel secondo libro delle Metamorfosi di Ovidio (II, vv. 760-801) - ricorrendo alla tradizione medico-biologica della teoria degli umori,16 che considera la gelosia un'affezione seconda, strettamente legata ad amore, generata, a seconda dei casi, da timore o invidia e, dunque, causa del 'gelo' intorno al cuore e del 'secco' nelle cavità cerebrali. E infatti Tasso, recuperando le riflessioni di Varchi, spiega che la casa della Gelosia è sulle montagne, circondata dalla neve

per dinotare la freddezza della sua natura, perché essendo ella timore, conviene che sia tale essendo ogni timor freddo perciò che il sangue si raccoglie e si raggela intorno al core. (ivi)

L'oscurità della caverna, invece, allude alla «mestizia di quei petti ov'ella alberga» (ivi) ed è femmina perché il temperamento delle donne è più freddo degli uomini. Infatti, 
[...] la fingono di sesso feminile, non tanto avendo riguardo a la voce, quanto a la proprietà delle donne; le quali essendo di temperatura di corpo fredde, e di spiriti più sottili, perciò più sottoposte al timore, e conseguentemente a la gelosia (ivi).

Anche Marino insiste sul carattere freddo della gelosia («pien di geloso e gelido desio [...] la cagion de' miei mali intento spio [...]»: MARINO 1653, 337), sulle sue origini infernali («peste d'Averno [...] non t'aborra anco l'inferno»: MARINO 1987, 193) e dedica al tema un ampio spazio nella sua produzione (PRANDI 1993). Si pensi, ad esempio, alla lunga apostrofe che apre il canto XII dell'Adone (1-6), che offre agli epigoni marinisti un'artificiosa summa di una topica ormai ben consolidata e che, non a caso, apre il ciclo narrativo di Falsirena, amante gelosa, sdegnosa, sofferente.

Margherita Costa dedica numerose liriche al topos dell'amante geloso e ha ben presente sia la disamina tassiana che l'accurata sintesi topica proposta da Marino. Rispetto ai versi dedicati alle manifestazioni della malattia d'amore, però, le liriche ascrivibili al tema della gelosia sono affidate sia ad una voce femminile che ad una maschile, che trova, infatti, maggior spazio ne Il violino. L'autrice, anche in questo caso, si appropria della tradizione lirica precedente e soprattutto di un topos che mantiene coerenza e unitarietà e che raggiunge proprio con Tasso e Marino il suo grado più alto di codificazione. La descrizione della gelosia, infatti, come individuato da Erika Milburn (2002) da cui riprendo le categorie che seguono, presenta alcune costanti tematiche e formali che possono essere rintracciate anche nei canzonieri costiani. Tra le immagini ricorrenti nella descrizione della gelosia ci sono riferimenti a veleni, serpenti, mostri mitologici; si allude ai suoi natali infernali e la sua presenza richiama sempre immagini di morte (proprio come nella lunga apostrofe in Adone XII):

$\begin{array}{ll}\text { serpenti e } & \text { O furia dell'Inferno, iniqua e ria, } \\ \text { veleni } & \text { o del mio Cor flagello, oltraggio e stento, } \\ & \text { o nemico di pace, o cruda arpia, } \\ & \text { o mio danno, o mio duolo, o mio tormento, } \\ & \text { o ghiaccio del mio foco, o morte mia, } \\ & \text { o veleno crudel del mio contento, } \\ & \text { o cruda fine del mio lieto stato, }\end{array}$


o pena senza colpa, o troppo ingrato.

(ott. 92, 6: CosTA 1638a, 371);

[...] Se tu mentisci il cor, mentisco il seno, se tu mi sai tradir, ti so tradire, s'assenzio tu mi dai, ti do veleno. [...]

(son. 54: ibidem, 454);

mostri

mitologici

origini

infernali

immagini

di morte
O Gelosia crudele, timor di gelo asperso ch'il bel sen del mio bene opprimi ogn'hor e con gelati e timorosi affetti tutto de' tuoi timor (cruda) l'infetti.

Ahi, lascia (mostro ingrato) disparger nel suo cuore dalla gelata bocca il tuo gelato e paventoso ardore. Ahi, che per te 'l mio Sole Eclissato si mostra.

(idill. 16, vv. 1-11: COSTA 1638b, 107);

Refiuto degl'Abissi, vivrò fra' miei dolori, fra' miei martiri e dissoluti Amori, e teco (Anima ingrata), che sei d'ogni mio mal cruda cagione, sarò qual cruda fera per tormentarti sempre, sarò tuo strazio eterno e tu solo sarai della mia Vita il disperato scherno. (idill. 15, vv. 113-22: ibidem, 106);

Ahi fera, ch'adogn'hora a morte mi condanni, e con le serpi dell'irsuto crine e visceri m'offendi e mi divori. (idill. 5, vv. 20-23: ibidem, 35);

Porta morte l'empio strale, l'alme uccideno i suoi vezzi, fugga, fugga ogni cor, fugga e lo sprezzi. (canz. 2, vv. 5-7: ibidem, 135). 
Tra i dispositivi retorici, invece, si individua un uso insistito di apostrofi, vocativi in apertura, anafore e antitesi. Di seguito, segnalo solo alcuni passi a scopo esemplificativo, perché questi tropi caratterizzano l'usus scribendi della poetessa e vanno ricondotti anche all'utilizzo implicito di strategie teatrali discusso sopra:

apostrofe

vocativo in apertura

Anafora
Iniqua / Fera spietata / Perfida / Crudele;

O fati, o Stelle, o del Celeste Impero numi pietosi $[\ldots]$

O gelosia crudele, timor di gelo asperso [...]

O Amore di te si duole ogni mentito amante [...];

Mi vedi abbandonata in queste piume, e quasi il spirto mio vedi spirante, mi vedi esangue e l'uno e l'altro lume mi vedi ogn'hor mancar'a te d'avante, mi vedi scaturir d'agl'occhi un fiume, mi vedi nel morir a te costante; e tu per secondare l'empio desire cerchi con doppio duol farmi morire. (ott. 66,5: CosTA 1638a, 255);

Dormo ne i miei dolor, ne i miei tormenti, dormo nel mio penar, nel mio martire, dormo ne i miei sospir, ne i miei lamenti, dormo ne i danni miei, nel mio languire, dormo a gl'affanni miei, a gli miei stenti, dormo nel cieco error del mio morire.

E dormo al fine addormentata ai vezzi del tuo finto scherzare a miei disprezzi. (ott. 75, 8: ibidem, 306);

Un affetto inusato, un pensier che m'uccide, un desir che m'offende, una fiamma che m'arde, un freno che m'accinge, un timor di timor così ripieno 
che mi fa mille volte mancar lo Spirto e 'l cor nel proprio seno.

(idill. 5, vv.13-19: CosTA 1638b, 34);

antitesi

Se ride la mia bocca, il cor si duole. se parla la mia lingua, l'alma tace. La fronte chiede guerra, il pensier pace. La verità smentisce le parole. Non crede ch'io non t'ami, o mio bel sole, il cor si finge un ghiaccio e in fuoco giace, finge sprezzar amore, d'Amor seguace, mostra fuggirti e te sol chiede e vuole. [...] (son. 31: CosTA 1638a, 431).

Inoltre, l'uso di antitesi (e di ossimori) ${ }^{17}$ serve a sottolineare la natura antitetica e ambigua dell'amore (GiGLIUCCI 2004). Il poeta, avvalendosi del contrasto tra guerra/pace, buio/luce o freddo/fuoco, usa un nucleo di immagini che servono a rappresentare l'esperienza del geloso, la cui mente oscilla sempre tra timore e sospetto, affetto e passione. Tra le antitesi messe a disposizione dalla tradizione lirica a cui attinge, Costa mostra una certa predilezione per la coppia fuoco/ghiaccio, una costante nelle descrizioni del contrasto d'amore (non solo quando il sentimento dominante è la gelosia). Nell'idillio 5, Donna tormentata dalla gelosia e infedeltà del suo amante s'uccide a da se stessa, raccolto ne Il Violino, la poetessa concentra tutte le immagini appena elencate nella forma del lamento elegiaco e in una struttura sintattica piana, ricca di domande retoriche, apostrofi e periodi incalzanti, adatta alla rappresentazione scenica:

\author{
Mostro dell'Inferno, \\ mostro spietato e rio, \\ qual novello veleno \\ di gelato timor mi serpi al core? \\ Qual nova e fredda cura \\ mi scorre in ogni vena? \\ Qual foco sotto il ghiaccio \\ de' tuoi pungenti strali \\ fa ch'io mi struggo e nell'ardor m'aghiaccio? \\ Ahi, che sento (infelice) \\ nell'Anima smarrita \\ un affetto inusato,
}


un pensier che m'uccide, un desir che m'offende, una fiamma che m'arde, un freno che m'accinge, un timor di timor così ripieno che mi fa mille volte mancar lo Spirto e 'l cor nel proprio seno.

Ahi, fera ch'ad ogn'hora a morte mi condanni, e con le serpi dell'irsuto crine le viscere m'offendi e mi divori.

Dalla gelata Bocca scocchi infausta favella ch'il Cor m'opprime e strugge; il perfido veleno ogn'hor l'alma m'accora ond'è forza (crudel) ch'io pera e mora. [...] (idill. 5, vv. 1-29: CosTA 1638b, 34-35).

Le conseguenze di questa affezione - si legge alla fine del componimento - sono portate all'estremo perché l'io lirico non trova soluzioni al suo dissidio e decide di togliersi la vita. In un altro idillio, la donna ingelosita soffre di un morbo generato da un «mortifero veleno» che opprime ogni senso, raggela il sangue e provoca dolore. Ma l'amato è sordo dinanzi a queste sofferenze e l'amante riconosce in un «pensiero insano, / [un] ostinato desire» la causa della sua sofferenza. Questa volta, però, non riesce a trovare conforto nella morte perché «l'anima disdegna / l'istesso Duce dell'oscuro Averno / e [la] disprezza (ahi lassa) ['] inferno» (idill. 15, 109-11: ibidem, 112). L'unico modo per superare l'impasse è trasformarsi in mostro crudele, assumerne le sembianze per tormentare e «lacerare» lo spietato cuore dell'amato. Nella folla dei gelosi, ancora, una bella donna maritata soffre per la gelosia del marito che le attribuisce colpe che non le appartengono, giudica con sospetto ogni gentilezza e vive sempre con il timore che lei possa tradirlo (idill. 16: ibidem, 107-13). Di contro, in un altro componimento, un amante si dichiara indifferente alla gelosia, non la odia come fanno tutti perché non è vittima di questa affezione, e soprattutto ha piena fiducia nell'amata, perché sa che i suoi occhi ghiacciano e feriscono coloro che osano guardarla (idill: 17: ibidem, 114-19). 
Il catalogo di belle donne e di amanti, dunque, presenta un altissimo livello di codificazione: immagini, strutture grammaticali e retoriche sono ripetute e portano all'esasperazione la formalizzazione letteraria della topica amorosa. In questo Costa non fa altro che ripetere la stessa operazione condotta dai lirici marinisti coevi e sono valide anche per lei le riflessioni di Giorgio Ficara:

l'amore, nel suo caratteristico oltranzismo intellettuale, viene ridimensionato e quasi ridotto in superficie dalla poesia di Marino e dei marinisti. Dopo l'alto cavillare di Petrarca e l'appassionato fantasticare dei neoplatonici, in questi prolifici canzonieri l'innamorato non sembra più nemmeno innamorato, ma un ilare notomista e un catalogatore avidissimo. Il pathos - il dramma iperbolico dell'innamoramento cede di fronte a un'altra iperbole: quella della variazione e dell'incostanza [...]» (FICARA 1993, 163).

La formalizzazione letteraria, dunque, prescinde dalla realtà e Costa non perde occasione di ribadirlo specialmente nei confronti del desiderio di morte, ridotto a convenzione retorica, ${ }^{18}$ perché «Troppo è cara la vita, e troppo costa, / e ben stolto è colui che non la stima» (ott. 82, 6 vv. 1-2: ibidem, 333). Tuttavia, la sofferenza per un amore è un sentimento reale che la poetessa conosce bene. Sotto la maschera di bella donna, infatti, non perde occasione per dispensare consigli alle giovani donne che si dona[no] troppo agli amori. Queste giovinette dovrebbero ascoltarla perché lei conosce bene le sofferenze d'amore e le false lusinghe, e spesso è stata vittima di un amante ingrato (ott. 34: ibidem, 147-57). Anzi, nonostante la mancata felicità che sempre accompagna una travolgente passione, come quelle di cui canta, non è possibile strapparsi ai lacci d'amore, anticipare le mosse del proprio amato, e non serve a nulla illudersi di conoscere le intenzioni e le passioni nascoste nel suo petto. In tal senso non sarà utile sperare, come suggeriva Momo, di aprire una finestra sul cuore, né sarà utile appropriarsi della vista potenziata offerta dal cannocchiale costruito da Galileo Galilei:

[...] e forse all'hora ti ricorderai del saggio detto, che già scrisse Momo che disse che già mai vedrassi un Core, senza fenestra, s'ama o scherne Amore. $[\ldots]$ 
Ch'a [il cuore] mille buchi e mille ripostini,

ha mille tane e sotterranei orrori, ha mille artigli et ha mille rampini, ha mille fabri per suoi finti amori, ha mille stipi, ha mille cassettini per conservarci i suoi mentiti ardori. E qual fenestra può esser bastante per scoprir ben di dentro un Core amante! Non basteria l'ochial del Galileo per vedergli suoi 'nganni e tradimenti; non è spirto sì scaltro o così reo che far ne possa mai veri argomenti; ha più lagrime in sé ch'il mare Egeo, non accoglie nel sen fiumi e torrenti. Ond'altro ci vorria ch'una apertura per scoprir in un Cor la finta arsura. (ott. 34, 11-14: ibidem, 150-51).

Mito e modernità, proprio come in Marino, si alleano in uno scopo comune, ma non sempre ottengono i risultati sperati. Le uniche armi di difesa verso la passione amorosa saranno pertanto il buon senso e l'amor proprio.

In un contesto tradizionalmente maschile e altamente formalizzato, Costa prova a rivendicare uno spazio di autonomia per le donne nella produzione poetica (pari all'autonomia e alla libertà guadagnate nella vita priva in quanto virtuosa, cantatrice $)^{19}$ e invita le giovinette che la ascoltano ad «amar con segno» e soprattutto ad «ama[r] chi più [le] par, ma più [se] stesse [...]» (ott. 34, 21: ibidem, 154).

«Donna son io che sol per ermonsura / mi diletto spiegar in queste rime / in varie forme l'amorosa arsura»: Margherita Costa emula del Marino

Dalla lettura di questi tasselli testuali si evince indubbiamente lo spesso sostrato mariniano della scrittura della virtuosa romana, già evidente dai titoli delle due raccolte qui esaminate. Costa ha letto Marino, canta i suoi versi ${ }^{20} \mathrm{e}$ recupera nei suoi canzonieri struttura e temi della Lira e della Sampogna. ${ }^{21}$ Infatti, le liriche sono incastonate in una fitta rete di legami intratestuali e intertestuali: elementi sintagmatici, paradigmatici e tematici compongono vari 
nuclei narrativi, messi in evidenza dagli argomenti autoriali posti in apertura di ogni lirica (come nei volumi del napoletano). Gli amanti sono co-protagonisti di una vicenda amorosa tormentata, fatta di soliloqui elegiaci e di dialoghi aspri e violenti, guidata da ira e vituperio; ma non mancano, come in Marino, scene sensuali ed erotiche. E soprattutto, la bella donna, in un evidente ribaltamento dei ruoli tradizionali, si fa interprete di un vero e proprio controcanto: l'io lirico, provato dalla lunga militanza amorosa, si mostra in più occasioni libero di rivolgersi direttamente all'oggetto del suo desiderio. Tuttavia, se la Lira detta la struttura e fornisce la materia amorosa (sdegno, gelosia, sofferenza per la lontananza, elogio della bellezza/bruttezza dell'amante, ecc.) e la Sampogna suggerisce l'uso della fortunata forma dell'idillio, ${ }^{22}$ è L'Adone il modello principale per la costruzione retorica del lamento amoroso di voce femminile. ${ }^{23}$ Mi riferisco, in particolare, all'episodio di Falsirena (canti XII-XIII) e alla sofferenza d'amore cui la maga è destinata. Gli elementi testuali, tematici e retorici del canto mariniano (ott. 129-292) sono, infatti, disseminati tra i versi dei due canzonieri: soliloquio e formulazione costante di domande retoriche [«Che miri? [...] O tua virtù che vale?» (MARINO 2013, ott. 175, 1253); «Ardo, lassa, o non ardo» (ibidem, ott. 198, 1263); «Forse amor? Non amor.» (ibidem, ott. 199, 1264); «Lassa, a qual cor parl'io se ne sono priva?» (ibidem, ott. 204, 1264); «Che ti val negar?» (ibidem, ott. 213, 1269)]; descrizione della natura ossimorica della passione amorosa [«Oimè ch'amor è foco / che 'nfiamma e strugge et io tremando agghiaccio» (ibidem, ott. 202, 1265); «sono trafitta e legata e 'nsieme accesa» (ibidem, ott. 203, v. 2); «Io vivo e moro pur» (ibidem, ott. 205, 1266)]; contrapposizione tra la forza travolgente dell'amore in giovane età e la saggezza della vecchiaia [si pensi alle posizioni contrastanti delle ancelle Idonia e Sofrosina, paragonate a «due fisici esperti / nel consiglio discordi» (ibidem, ott. 238, 1279)]. Insomma, i soliloqui di Falsirena e della bella donna sono entrambi costruiti su dispositivi antitetici e iterativi e sono modulati su una successione di formule interrogative e di sillogismi, utilizzate come strumenti per superare la malattia, razionalizzare e accettare il sentimento che le sconvolge. Come mostrano questi esempi, i debiti contratti verso il poeta napoletano, dunque, sono numerosi, non si limitano a La Chitarra e a Il Violino e meritano ulteriori indagini. ${ }^{24}$ 
Questi procedimenti retorici, inoltre, sono largamente utilizzati nella musica monodica e melodrammatica per la costruzione di arie affidate alle virtuose; anzi, l’inserimento del lamento in un dramma per musica mira a mettere in scena la virtuosità della cantante protagonista. In aggiunta, le tematiche amorose declinate dall'autrice affollano i repertori delle cantate romane. Dunque, la scelta di questo modello non va ricondotta alla sola consonanza di genere (i lamenti e i tormenti di Falsirena hanno valore universale e possono essere utilizzati da una donna per raccontare le traversie di un'altra donna), ma anche alla vicenda biografica di Costa. Infatti, alcune ottave del lamento (Adone, XII, 198-207) appaiono nelle Musiche a due voci di Sigismondo d'India pubblicate nel 1615 (VASSALLI 1989) ed entrano subito nel repertorio lirico delle cantatrici; e, quindi, non è affatto improbabile che la poetessa si sia esercitata o esibita nel loro canto. Inoltre, Costa fu coinvolta in una scommessa canora insieme a Cecca del Padule ${ }^{25}$ per interpretare Falsirena - appunto - ne $L a$ Catena d'Adone (1626), favola boschereccia di Ottavio Tronsarelli musicata da Domenico Mazzocchi, il cui ipotesto è costituito dai canti XII e XIII del poema mariniano (SANTACROCE 2014; GIGLIUCCI 2015).

Giocando con la sensibilità del lettore coevo, che riconosce immediatamente l'intertesto, Costa è una poetessa marinista, mostra un riuso intelligente del modello, lo emula, desidera eguagliarlo e superarlo, portandolo però nella propria sfera di competenza: il lamento elegiaco cantato $^{26}$. Pensate per la pagina scritta e come strumento di autopromozione, queste liriche sono costruite su temi e moduli propri della poesia per musica, ${ }^{27}$ per la messa in scena sotto forma di arie e/o recitativi, per dimostrare la virtuosità della loro interprete. La poetessa virtuosa, infatti, dà voce ad un io lirico femminile teatralizzato in volumi che nella loro struttura ripetitiva e uniforme sembrano pagine di un personale canovaccio ${ }^{28}$ di un repertorio lirico e musicale da usare all'occorrenza per dimostrare il proprio valore non solo di cantante, e dunque di interprete, ma anche di autrice.

Insomma, se appropriandosi di stili, immagini, forme metriche, strutture retoriche ed espressioni lessicali proprie di Marino, della lirica e della musica coeva, Costa non aggiunge nessun elemento innovativo alla secolare tradizione della lirica amorosa, rispetto ai modelli, però, ci sono due elementi di novità che 
rendono unica questa sua produzione marinista e vanno ricondotti alla sua esperienza biografica: da un lato la teatralità implicita delle liriche che deriva dal mestiere di virtuosa; dall'altro la rifunzionalizzazione al femminile del messaggio d'amore in campo letterario. Ripetendo fino allo sfinimento la messa in scena della stessa situazione, Costa insiste sulla funzione espressiva della comunicazione, generalmente demandata all'uomo e, giocando con le aspettative gender del lettore (abituato a questo continuo e fluido scambio di voci solo nelle cantate e non sulla pagina scritta), procede in un'inversione dei ruoli del mittente e del destinatario per donare, in un contesto misogino, una voce femminile alle sue sodali, con versi che definisce, ironicamente e secondo i moduli dell'excusatio, nel capitolo d'apertura de La Chitarra

[...] schietti e mal vestiti ricchi d'errori e poveri di merto di rozzo stile e poco ripoliti (cap. vv. 75-77: CosTA 1638a, 4). 
* I testi riprodotti non godono di un'edizione moderna e la trascrizione semidiplomatica è stata condotta secondo i seguenti criteri: sono stati adeguati all'uso moderno la separazione delle parole, la punteggiatura e i segni diacritici; è stato normalizzato l'uso delle doppie; si è disciplinato l'uso sovrabbondante delle maiuscole, conservandole solo nei nomi propri, nei titoli onorifici o laddove specificassero un significato metaforico o traslato.

${ }^{1}$ Margherita Costa ha all'attivo sei canzonieri: La Chitarra (1638), Il Violino (1638), Lo Stipo (1639), La Selva di cipressi (1640), La Selva di Diana (1647) e La Tromba di Parnaso (1647). In queste raccolte, si leggono liriche di ispirazione elegiaco-amorosa, epinici, epicedi e poemetti burleschi. Una rassegna della produzione costiana si legge in COSTA-ZALESSOW 2015, 19-45. Per il profilo dell'autrice si veda il lungo, seppur datato, contributo di BIANCHI 1924-1925. Si vedano anche GOETHALS 2017 e COSTA 2018, 1-73.

${ }^{2}$ Le due raccolte possono essere lette globalmente perché si presentano come «un singolo libro di poesie, caratterizzato [...] da ridondanza tematica ed equilibrio strutturale [...] un libro che rivela alla lettura sia una relazione semantica e strutturale assai stretta tra i suoi componenti, sia un principio e un fondamento interni» (TESTA 1983, 20).

${ }^{3}$ Le false indicazioni editoriali (Francfort, Daniel Wastch) possono essere ricondotte ad un travestimento tipico della pratica attoriale, perché i comici e le comiche dell'arte tendevano a pubblicare i propri testi in vesti editoriali false. Va ricordato che la prima professione di Costa è il canto: è una donna di scena, una cantante di camera, e usa questa strategia per ingraziarsi le simpatie del pubblico (e soprattutto dei suoi committenti) e dimostrare di essere famosa oltre i confini delle corti fiorentina e romana in cui lavora. Invece, la pubblicazione delle due raccolte va attribuita alla bottega Fiorentina di Amadore Massi e Lorenzo Landi, come dimostrato dall'indagine sull'apparato paratestuale dei due volumi condotto da Julie Robarts: «The definitive proof, however, of Florentine printing of La chitarra and Il violino by Massi and Landi is found in the decorative woodblocks that mark the sections of the text in Il violino, containing the printer's initials "A.M." - Amadore Massi - nestled in the topmost curved pendants, left and right» ROBARTS $(2019,81)$.

${ }^{4}$ L'ottava resta il metro predominante anche nelle raccolte successive. Ne Lo Stipo, le 45 canzoni in ottava rima sono intervallate da un epigramma latino e dalla sua traduzione in endecasillabi sciolti. Le 14 liriche de La Selva dei cipressi sono canzoni in ottave. Nelle ultime due raccolte, invece, si registra la superiorità del sonetto rispetto all'ottava: 30 sonetti e 15 ottave ne La Selva di Diana; 28 sonetti, un madrigale e 12 ottave ne La Tromba. La predilezione per questa forma metrica trova spiegazione nella carriera di virtuosa di Costa e nella fortuna della pratica dell'intonazione cantata in ottava rima; una forma che gode di una lunga storia nella tradizione letteraria e musicale peninsulare. Maurizio Agamennone a tal proposito scrive: «[...] il vero motivo del "successo" del metro e del modello formale sta proprio in questa fruibilità agevole ed estesa, nella fluida versatilità che è riuscita ad alimentare efficacemente istanze e necessità espressive assai mutevoli, prestandosi altrettanto bene sia alla conduzione di lunghe e complesse saghe narrative, con decine e decine di stanze coerentemente integrate in azioni performative lunghe e spettacolari, sia alla elaborazione estemporanea di concetti astratti e complessi per una estensione temporale intermedia, sia all'espressione di sentimenti più intimi e personali, in forme e tempi assai più contenuti» (AGAMENNONE 2017, XIII; corsivo mio). Va poi segnalata la fortuna goduta dall'ottava per musica nella corte toscana di Ferdinando II, dimostrata dai manoscritti riccardiani di arie per musica in ottava rima, nei quali «il soggetto prevalente è l'amore non corrisposto, con una preferenza per le metafore fiamma d'amore / fiamma infernale, donna crudele / falco, usignolo / amante» (TOMASELLO 2003, 120) a cui si aggiunge il canto dell'amante geloso, rimodulato sulle descrizioni madrigalistiche di Battista Guarini. Questi temi e queste immagini sono presenti in tutto il corpus di Margherita Costa. 
${ }^{5}$ La caratterizzazione dell'amante di sdegno è in linea con la definizione di tale sentimento riportata nella voce del Vocabolario della Crusca. La prima (1612) e la seconda edizione (1623) definiscono lo sdegno come «ira, cruccio, indegnazione». Segnalo, inoltre, che nella terza edizione (1691) il campo semantico si allarga anche al disprezzo. Un ciclo dello sdegno si legge nelle rime di Tasso (TOMASI 2012) e in Tansillo (MILBURN 2003, 84-107). Besomi e Martini segnalano una serie di componimenti di sdegno nel Commento al sonetto 76 del Cavalier Marino in MARINO 1987, 186. Il tema conosce una discreta fortuna anche in musica (si pensi, ad esempio, a GIGLI 1585).

${ }^{6}$ Già Gaspara Stampa aveva messo al centro del suo canzoniere il lamento elegiaco della donna innamorata di un amante assente. Le sue Rime, però, perfettamente inserite nel canone petrarchesco in cui vengono elaborate, hanno «un valore esemplare [...] sono un doloroso bilancio di un'esperienza amorosa, hanno un valore didattico» (TARSI 2015, 19). Costa, invece, rispetto alla padovana non attribuisce nessun valore didascalico ai suoi versi. La predominanza del lamento è una forma di espressione del virtuosismo drammatico.

${ }^{7}$ Il trattato, scritto fra il 1276 e il 1286, circola in forma manoscritta per più di due secoli fino alla sua prima edizione a stampa pubblicata a Lione nel 1504, che ne determina la fortuna lungo tutto il XVI secolo.

${ }^{8}$ Nella Chitarra la superiorità numerica di componimenti con io lirico femminile è plateale. Presentano un io lirico maschile solo quattro testi su 225 (sono esclusi in questo elenco e anche dalla tabella 1 I Caramogi che chiudono il volume): il canto 42 Virtuoso innamorato di Bella Donna (Costa 1638a: 184-85) e 44 Virtuoso di nuovo a Bella donna (ivi, 188-89), che fanno parte di una tenzone per le rime; il componimento 57 Uno amante ch'alluscir d'una devozione manda a donare un fiore ad una giovane per un povero (ivi, 227-28) e il sonetto 81 Uno amante partito dalla sua donna havendo detto male di lei per conseguire l'amore di altra donna si duole del suo errore (ivi, 481). Nel rapporto donna/uomo le liriche de Il Violino sono invece nettamente sbilanciate sul secondo versante: sei idilli e diciannove canzonette sono affidate ad una voce maschile. Anche in questo caso, la seconda raccolta sembra colmare i vuoti della prima.

${ }^{9}$ «Non amo e sono Amante, ardo e non godo, / provo mille martir, non sento ardore, / cinta mi veggio e pur non scorgo il nodo; / piango mia libertà, sciolta d'amore / le mie miserie in altra lingua snodo, / tengo giaccio nel sen, foco nel Core / e vaga all'altrui duol, nel duolo io vivo, / ho penzier fermo e desir fugitivo» (ott. 24, 4: ivi, 110-12).

${ }^{10}$ La citazione è tratta dal sonetto $O$ gelosia, d'amanti orribil freno di Iacopo Sannazzaro (vv. 10-11).

${ }^{11}$ La prosopopea della Gelosia, ad esempio, viene utilizzata nel Prologo di molte commedie. Si pensi a La Fantesca di Giovan Battista della Porta (REFINI 2006, 74-79). Oltre ad aprire le azioni sceniche, la gelosia è al centro di numerose commedie e favole pastorali pubblicate nel XVII secolo.

${ }^{12}$ La trattatistica amorosa, che è sempre in dialogo con quella medica e filosofica, ne riconosce di due tipi: una gelosia dannosa, associata al rapporto coniugale, e una buona, legata alla figura dell'amante cortese. Sull'argomento si veda FAVARO 2012.

${ }^{13}$ Si pensi ad esempio alla Lettura sopra un sonetto della Gelosia di Mons. Della Casa di Benedetto Varchi (VARCHI 1560; TOMASI 2019), alla Lezione della gelosia letta nella Academia fiorentina di Niccolò Vito (VITO 1585) o Il Cataneo overo della gelosia di Ludovico Zuccolo (ZUCCOLO 1615).

${ }^{14}$ Pronunciato a Modena nel 1577, il Discorso fu pubblicato nel 1585 a Venezia da Aldo Manuzio. L'autore presenta lo stesso punto di vista dei trattati d'amore del Cinquecento perché propone una visione negativa della gelosia, la stessa declinata anche nelle ottave Sulla gelosia. Completamente opposta è invece l'idea proposta ne Il forastiero napoletano ovvero la Gelosia. Qui Tasso, basandosi sulla retorica di Aristotele, prova a rivalutare il giudizio sulla gelosia, la innalza a virtù e le riconosce un ruolo fondamentale nella creazione poetica perché è la condizione che permette al poeta di parlare d'amore. Ad esempio, a proposito di Ariosto dichiara: «F.N. Molte cose e tutte ree accompagna insieme questo famoso poeta in biasimo e in vituperio de la gelosia; ma debbiam noi credere quel ch'egli dice? C.C. Egli fu non solamente gran poeta, ma ancora grande innamorato: laonde ragionando egli de le amorose passione, se gli dee prestar credenza» (TASSO 2018, 53). 
${ }^{15} \mathrm{Gli}$ affetti sono determinati dalla combinazione dei quattro umori vitali del corpo (sangue, bile gialla, bile nera, flegma), corrispondenti ai quattro elementi (aria, fuoco, terra, acqua) e alle quattro qualità elementari (caldo, secco, freddo, umido). Un rapporto equilibrato tra gli umori del corpo tende verso una condizione di salubre armonia; il loro squilibrio, invece, conduce verso una disarmonia patologica. Va ricordato che nei primi decenni del XVII secolo, la teoria umorale si lega profondamente alla teoria musicale. Infatti, medici e musicisti sostengono che le qualità melodiche e le armonie di una composizione musicale influiscono, tramite l'udito, sull'equilibrio umorale e possono descrivere i contenuti espressivi del testo poetico col fine di muovere gli affetti (DONÀ 1967).

${ }^{16}$ L'associazione gelosia-gelo è un'immagine che colpisce immediatamente il lettore moderno, solito accomunare questa passione all'ardore e al caldo. Invece, tale connotazione affolla i volumi sull'argomento, nei quali è giustificata la natura fredda, è spiegato il sistema metaforico legato al ghiaccio e al freddo e l'affezione è ricondotta alla teoria galenica degli umori: «Et che il gielo si metta per la paura, cioè l'effetto per la cagione è figura usitatissima non solo appresso i dicitori in rima, ma eziando a quelli di prosa: la cagione perché chi teme diventi pallido e freddo è perché contrae e debilita il cuore. Onde la natura per soccorrerlo (essendo il cuore il più nobile membro dell'uomo) come quello che secondo i Peripatetici è il primo a nascere e l'ultimo a morire, vi manda il sangue della parte di sopra e non bastando questo vi manda anco in suo aiuto di quello di sotto e di qui nasce la pallidezza e 'l gielo, tremasi poi perché tremando il cuore trema dietro il suo moto tutto 'l corpo» (VARCHI 1560, 95v).

${ }^{17}$ Valgono per Costa le riflessioni condotte da Andrea Battistini sul gioco antitetico offerto da Marino: «In questo senso è lecito affermare che l'antitesi ha nell'Adone una valenza ossimorica, nel senso che una componente non si annulla nell'altra, ma si somma, proprio come in un'ellissi un fuoco non si sovrappone mai all'altro né vi si identifica ma crea con l'altro una tensione dovuta alla compresenza [...]» (BATTISTINI 2007, 174-75).

${ }^{18}$ «E proprio uso in amor di mille amanti / il dir voglio morir, voglio mancarmi, / e sparger coi sospiri mentiti pianti / e dir per amor tuo voglio piagarmi. / Ma s'il morir si desse per contanti, / nessuno diria per te vuoglio ammazzarmi; / il dir per te mi moro è sciocco detto / d'un semplice parlar senza altro effetto» (ott. 82, 9: CosTA 1638a, 336).

${ }^{19} \mathrm{Si}$ pensi, ad esempio, alla vicenda biografica della sorella Anna Francesca e all'impresa dell'Ergidoro (MEGALE 1996).

${ }^{20}$ Sulla fortuna del Marino in ambito musicale resta un valido punto di partenza SimONDGIDROL 1973. Si veda anche BIANCONI 1991, 9-16 e MAZZOTTA 2013. Un lungo elenco di tutti i versi del napoletano messi in musica è consultabile nel catalogo RePIM (http://repim.muspe.unibo.it/risultati.aspx). Si veda, infine, il recente contributo di GiLES 2020.

${ }^{21}$ Le opere di Costa sono molto vicine a Marino in termini di ispirazione e di struttura. Ad esempio, costruisce Il Violino secondo la tecnica del technopaegnion usato dal napoletano ne La Sampogna (MARINO 1993).

${ }^{22} \mathrm{La}$ struttura dell'idillio costiano segue quella dell'idillio mariniano. La 'selva' di endecasillabi e settenari, infatti, è divisa in strutture binarie, ternarie o quaternarie, presenta simmetrie retorico-sintattiche, gruppi di rime baciate e rime interne, e uso dell'assonanza e della consonanza. Tutte le strategie retoriche, come è evidente in questo idillio, mirano ad attribuire al verso un'intonazione drammatica. Per una precisa descrizione delle scelte metriche mariniane, contenitore in cui Costa attinge, si veda la Nota metrica in SALVARANI 2006, CIIICXXVIII.

${ }^{23}$ Nella struttura e formulazione del lamento amoroso, Costa ha presente anche le stanze dedicate a Lidia abbandonata pubblicate nella sezione Capricci della terza parte della Lira: «[...] O crudel, quanto bello a gli occhi miei / già parte al tuo patir. Ma che cur'io / perdere il cor se te mio cor perdei? [...]» (10); «Lassa e dritto sia ben, ch'io con le stelle / qui lagnandomi sola e con l'arene [...]» (23); «Misera, che vaneggio? E dove sono?» (27) in MARINO 1629, 27990.

${ }^{24}$ A mio avviso, Costa si ispira al canto XII dell'Adone anche per l'architettura concettuale de Lo Stipo. Il volume è presentato come un grande mobile d'uso domestico, nei cui cassetti sono riposti pietre e metalli preziosi: 1. carbunculi e diamanti; 2 . smeraldi e rubini; 3. oro e perle; 4. ametiste, lapislazzuli e turchine; 5 . ambre, coralli e zaffiri; 6 . alabastri, cristallo di 
Boemia e gioie false; 7. grisoliti e giacinti. Un elenco delle stesse pietre si legge in Adone, XII 163-67 (MARINO 2013, 1249-50).

25 «[...] nel 1626 i principi Giandomenico Lupini e Giovanni Giorgio Aldobrandini decisero di rendersi conto quale delle loro fosse la cantante migliore ed escogitarono l'idea di farle apparire entrambe nella stessa opera. A quel tempo erano già al servizio dell'Aldobrandini il poeta Ottavio Tronsarelli e il compositore Domenico Mazzocchi, i quali esaudirono la richiesta di fornire un lavoro adatto a questa specie di gara con La Catena d'Adone, basata su una sezione del famoso poema del Marino. Le due cantanti concorrenti, ugualmente famose e non soltanto per le loro doti musicali, erano Margherita Costa, sostenuta dal Lupini, e Francesca (conosciuta anche col diminutivo di 'Cecca') del Padule [...], favorita dell'Aldobrandini. L'aspettativa di una serata emozionante spinse il Marchese Evandro Conti ad offrire il suo palazzo per la gara, ma il tutto fu ostacolato dalla moglie dell'Aldobrandini che riuscì ad evitare un probabile scandalo facendo sostituire le due donne con due castrati» (GIANTURCO 1982, 212).

${ }^{26}$ Sulla fortuna e la struttura del lamento in musica nel XVII secolo (e le sue relazioni con il femminile) si veda il recente contributo di REFINI 2020.

${ }^{27}$ È indubbia la profonda consonanza tematica (e metrica) tra la produzione di Costa e la poesia per musica coeva, specialmente con gli attribuiti propri delle cantate romane (MORELLI 2006; JEANNERET 2013). Ad esempio, il ms. Bar. Lat. 4175 (XVII sec.), conservato presso la Biblioteca Apostolica Vaticana, presenta una raccolta di ariette per musica i cui temi - gelosia, sofferenza d'amore, lamento della donna abbandonata, crudeltà dell'amante etc. - sono declinati nei due canzonieri costiani. Nel ms. Chig. Q.VIII.177 (simile per struttura e temi al barberiniano), invece, è presente una cantata attribuita dal copista in una nota a margine dello spartito «alla sig. Costa» (cc. 15v-18r). O Dio, voi che mi dite è una cantata per due voci, a contralto e soprano, con basso continuo e con musiche di Marco Marazzoli (ringrazio Ivana Zaurino per la lettura dello spartito e le indicazioni tecniche). Tenderei, però, ad attribuire il testo ad Anna Francesca (e non a Margherita come fa CosTA-ZALESSOW 2015, 45) perché lavora a stretto contatto con il musicista parmense (autore, ad esempio, delle musiche de Il giudizio della ragione e de l'Egisto).

${ }^{28}$ Le due raccolte, insieme a Lo Stipo, costituiscono una sorta di canovaccio del faire poétique costiano: temi, elementi narrativi, lessico, espressioni e architettura retorica verranno riutilizzati dalla poetessa in tutte le opere successive. Mi limito a riportare pochi esempi tratti da testi che hanno finalità dichiaratamente teatrali: ne La Flora feconda. Dramma I, 1, i lamenti di Zefiro e Flora sono modellati sui lamenti che si leggono ne Il Violino: «ZEF. Ah Flora, amato bene, / luce del viver mio, / ristoro del mio danno, / pace del mio gioir, / scopri a me 'l tuo martoro / che, se più taci, io moro» (CosTA 1640, 3); ne Li Buffoni II, 8 (ringrazio Jessica Goethals per la segnalazione), Tedeschino e Marmotta parlano d'amore in termini di malattia: «TED. Febre maligna de la vita mia, / petecchia del mio volto, mio dolore, / de la gola catarro e schinanzia [...]» (Costa 1641, 119; Costa 2018); ne Gli amori della luna I, 4, Diana rivolge una feroce invettiva contro l'amato Endimione: «DIA. Ah crudo, e pur non senti / ne l'agghiacciato petto, / qual già sentir solevi, / di focose faville ardente affetto? / Non così mi dicevi / quando ritrosa anch'io / havea ghiaccio il desio [...] E hor fra tanto gelo / neghittoso ti stai / e volgi a me sol per schernirmi i rai» (COSTA 1654, 26). 


\section{Bibliografia}

AGAMENNONE 2017:

Maurizio AGAMENNONE, Cantar ottave. Un'introduzione, in Cantar ottave. Per una storia culturale dell'intonazione cantata in ottava rima, a c. di Id., Lucca, Libreria Musicale Italiana, 2017, pp. VII-XXVI.

\section{BATTISTINI 2007:}

Andrea BATTISTINI, "Paradiso infernal, celeste inferno». Ossimori d'amore nell'Adone di Giovan Battista Marino, in «RiLUne», 7 (2007), pp. 171-87.

BIANCHI 1924-1925:

Dante BIANCHI, Una cortigiana rimatrice del Seicento: Margherita Costa, in «Rassegna critica della letteratura italiana», XXIX (1924), pp. 187-203 e XXX (1925), pp. 158-211.

\section{BIANCONI 1992:}

Lorenzo BiAnconi, G. B. Marino e la poesia per musica, in Id., Il Seicento, Torino, EDT, 1992, pp. 9-16.

\section{CHERCHI 1992:}

Paolo CHERCHI, A Dossier for the Study of Jealousy, in Eros and Anteros. The Medical Traditions of Love in the Renaissance, a c. di Donald Beecher and Massimo Ciavolella, University of Toronto Italian Studies, Dovehouse Editions 1992, pp. 12334 .

Ciavolella 1976:

Massimo Ciavolella, La malattia d'amore dall'antichità al Medioevo, Roma, Bulzoni, 1976.

CosTA 1638a:

Margherita CosTA, La Chitarra, Francoforte, Daniel Watsch, 1638.

COSTA 1638b:

Margherita CosTA, Il Violino, Francoforte, Daniel Watsch, 1638.

CosTA 1640:

Margherita Costa, Flora feconda. Dramma, Firenze, Massi\&Landi, 1640.

CosTA 1641:

Margherita CosTA, Li Buffoni, Firenze, Massi\&Landi, 1641.

CosTA 1654:

Margherita Costa, Gli amori della luna, Venezia, Giuliani, 1654.

COSTA-ZALESSOW 2015:

Natalia Costa-ZALESSOW, Voice of a Virtuosa and Courtesan. Selected poems of Margherita Costa, New York, Bordighera Press, 2015. 
COSTA 2018:

Margherita CosTA, The buffoons: a ridiculous comedy. A bilingual edition, a c. di Sara E. Díaz, Jessica Goethals, Toronto, Arizona Center for Medieval and Renaissance Studies, 2018.

D'AMICO 2005:

Silvia D'AMICO, Le Discorso della gelosia de Torquato Tasso: la doxa d'une passion in XVI siecle entre medecine et tradition litteraire, in La transmission des savoirs au Moyen Age et à la Renaissance, a c. di Franck La Brasca, Alfredo Perifano, Besancon, Presses universitaires de Franche-Comte, 2005, pp. 59-74.

DE LISO 2020:

Daniela DE LISO, Le lettere amorose di Margherita Costa, in (Auto)narrativas: hacia la construcción de un canon alternativo en italiano, a c. Sara Velázquez García e Laureano Núñez García, Salamanca, Ediciones Universidad de Salamanca, 2020, pp. 47-64.

DI MARO 2019:

Maria Di MARO, Il Violino di Margherita Costa: prime indagini, in Escritoras italianas inéditas en la querella de las mujeres: traducciones en otros idiomas, perspectivas y balances, a c. di Salvatore Bartolotta e Mercedes Tomo-Ortiz, Madrid, UNED editorial, 2019, vol. II, pp. 43-56.

DI MARO 2020:

Maria DI MARO, Una poetessa del XVII secolo: Margherita Costa, in (Auto)narrativas: hacia la construcción de un canon alternativo en italiano, a c. di Sara Velázquez García e Laureano Núñez García Salamanca, Ediciones Universidad de Salamanca, 2020, pp. 81-97.

DONÀ 1967:

Mariangela DonÀ, Affetti musicali nel Seicento, in «Studi Secenteschi», I (1967), pp. 75-94.

FAVARO 2012:

Maiko FAVARO, L'ospite preziosa: presenze della lirica nei trattati d'amore del Cinquecento e del primo Seicento, Lucca, Maria Pacini Fazzi, 2012.

FICARA 1993:

Giorgio FICARA, Solo come un Adone, in Solitudini. Studi sulla letteratura italiana dal Duecento al Novecento, Milano, Garzanti, 1993, pp. 148-74.

GDLI:

Grande dizionario della lingua italiana, a cura di Salvatore Battaglia, Torino, UTET, 2003, vol. XXI.

GIANTURCO 1982:

Carolyn GIANTURCO, Nuove considerazioni sul «tedio del recitativo» delle prime opere romane, in «Rivista italiana di Musicologia», XVIII, 1982, pp. 212-39.

GIGLI 1585:

Giulio GIGLI, Sdegnosi Ardori. Musica di diversi auttori, sopra un istesso soggetto di parole, Monachi, excudebat Adamus Berg, 1585. 


\section{GIGLIUCCI 2004:}

Roberto GIGLIUCCI, Contraposti: petrarchismo e ossimoro d'amore nel Rinascimento: per un repertorio, Roma, Bulzoni, 2004.

\section{GIGLIUCCI 2015:}

Roberto GIUGLIUCCI, Tronsarelli e la Catena d'Adone fra morte di Marino e messa all'indice del poema, in «Studi (e testi) italiani», 36 (2015), pp. 45-54.

GILES 2020:

Roseen Giles, Giambattista Marino's L'Adone: A Drama of Madrigals, in «The Italianist», 40 (2020), 3, pp. 419-40.

GOETHALS 2017:

Jessica GoETHALs, The Bizarre Muse: the literary Persona of Margherita Costa, in «Early modern women», 12 (2017), 1, pp. 48-72.

\section{JEANNERET 2013:}

Christine Jeanneret, Gender ambivalence and the expression of passions in the performances of early Roman cantatas by castrat and female singer, in The emotional power of music: multidisciplinary perspectives on musical arousal, expression, and social control, a c. di Tom Cochrane, Bernardino Fantini e Klaus R. Scherer Oxford, Oxford University Press, 2013, pp. 85-101.

MARINO 1629:

Giovan Battista MARIno, Della Lira del Cavalier Marino. Parte terza, Venezia, Ciotti, 1629.

MARINO 1657:

Giovan Battista MARINO, La Lira, Venezia, per Francesco Baba, 1653.

MARINO 1987:

Giovan Battista MARINO, Rime amorose, a c. di Ottavio Besomi e Alessandro Martini, Modena, Panini, 1987.

MARINO 1993:

Giovan Battista MARINO, La Sampogna, a c. di Vania De Maldè, Parma, Fondazione Pietro Bembo/Ugo Guanda editore, 1993.

MARINO 2013:

Giovan Battista MARINo, Adone, a c. di Emilio Russo, BUR, Milano, 2013, voll. II.

MARTINI 1984:

Alessandro MARTINI, Amore esce dal Caos. L'organizzazione tematico-narrativa delle rime amorose del Tasso, in «Filologia e critica», IX (1984), pp. 78-121.

\section{MARTINI 2002:}

Alessandro MARTINI, Le nuove forme del Canzoniere, in I capricci di Proteo: percorsi e linguaggi del barocco. Atti del Convegno internazionale di Lecce, 23-26 ottobre 2000, Roma, Salerno editrice, 2002. 
MAZZOTTA 2013:

Giuseppe MAZzOTTA, Marino's Operatic Esthetics, in Word, Image, and Song. Essays on Early Modern Italy, a c. di Rebecca Cypress, Beth L. Glixon e Nathan Link, Rochester, University of Rochester Press, 2013, pp. 251-64.

MEGALE 1996:

Teresa MEgale, Il principe e la cantante: riflessi impresariali di una protezione, in «Medioevo e Rinascimento», VI (1996), n. s. III, pp. 221-33.

MICALI 2007:

Simona Mical, Gelosia, in Dizionario dei temi letterari, a c. di Remo Ceserani, Mario Domenichelli e Pino Fasano, Torino, UTET, 2007, vol. II F-O, pp. 967-75.

MILBURN 2002:

Erica MilbuRn, D'Invidia e d'Amor figlia si ria: Jealousy and the Italian Renaissance Lyric, in «The Modern Language Review», 97 (2002), 3, pp. 577-91.

MILBURN 2003:

Erica MilbuRn, Luigi Tansillo and Liric Poetry in Sixteenth-Century Naples, Leeds, Maney Publishing for the Modern Humanities Research Association, 2003.

MORELLI 2006:

Arnaldo MORELLI, "Perché non vanno per le mani di molti...». La cantata romana del pieno Seicento: questioni di trasmissione e di funzione, in Musica e drammaturgia a Roma al tempo di Carissimi, a c. di Paolo Russo, Venezia, Marsilio, 2006, pp. 21-39.

PERI 1996:

Massimo PERI, La medicina dei poeti e la poesia dei medici, Soveria Mannelli, Rubettino, 1996.

POMA 2007:

Roberto Poma, Metamorfosi dell'hereos. Fonti medievali della psicofisiologia del mal d'amore in età moderna (XVI-XVII), in «RiLUne», 7 (2007), 2, pp. 39-52.

PRANDI 1993:

Stefano PRANDI, Marino, Tasso e la Gelosia, in «Filologia e Critica», XVIII (1993), pp. 114-21.

PRANDI 2014:

Stefano PRANDI, Variazioni tassiane sul tema della Gelosia, in «Quasi ombra e figura de la verità». Pensiero e poesia in Torquato Tasso, Padova, Antenore, 2014, pp. 30-51.

\section{REFINI 2006:}

Eugenio REFINI, Prologhi figurati: appunti sull'uso della prosopopea nel prologo teatrale del Cinquecento, in «Italianistica: rivista di letteratura italiana», 35 (2006), 3, settembre/dicembre, pp. 61-86.

REFINI 2020:

Eugenio REFINI, 'Parole tronche et imperfette': The Lament as a 'Mode' across Poetical and Musical Genres, in «The Italianist», 40 (2020), 3, pp. 441-62. 
ROBARTS 2019:

Julie L. ROBARTS, Challenging Male Authored Poetry: Margherita Costa's Marinist Lyrics (1638-1639), Tesi di dottorato, The University of Melbourne, Australia, 2019.

SALVARANI 2006:

Giovan Battista Marino, La Sampogna, a c. di Marzio Pieri, Alessandra Ruffino, Luana SALVARANI, Trento, la Finestra, 2006.

SANTACROCE 2014:

Simona SANTACROCE, «La ragion perde ove il senso abonda». La catena d'Adone di Ottavio Tronsarelli, in «Studi secenteschi», LV (2014), pp. 135-53.

SIMON-GIDROL 1973:

Roger Simon, D. Gidrol, Appunti sulle relazioni tra l'opera poetica di G. B. Marino e la musica del suo tempo, in «Studi secenteschi», XIV (1973), pp. 81-187.

STUMPO 1996:

Irene Cotta STUMPO, de Medici Ferdinando, in Dizionario biografico degli italiani, vol. 46, 1996, ad vocem.

TARSI 2015:

Maria Chiara TARSI, La poesia di Gaspara Stampa e la tradizione elegiaca, in «Testo», 69 (2015), 1, pp. 7-28.

TASSO 1875:

Torquato TASSO, DISCORSO SULLA GELOSIA, in Le prose diverse, a c. di Cesare Guasti, Firenze, Le Monnier, 1875, vol. II, pp. 171-85.

TASSO 2018:

Giacomo Vagni, Federica Alziati, Torquato TASso, Dialoghi: Il Forestiero Napolitano overo de la gelosia e Il Gianluca overo de le maschere, in «Per Leggere», 35 (2018), pp. 43-91.

TESTA 1983:

Enrico TESTA, Il libro di poesia: tipologie e analisi macrotestuali, Genova, Il Melangolo, 1983.

TOMASELLO 2003:

Santina TOMASELlo, Arie per cantar l'ottave ceciliane nei manoscritti riccardiani, in Rime e suoni alla spagnola. Atti della Giornata Internazionale di Studi sulla Chitarra Barocca. Firenze, Biblioteca Riccardiana, 7 febbraio 2002, a c. di Giulia Veneziano, Firenze, Alinea 2003, pp. 109-37.

TOMASI 2012:

Franco TOMASI, Lettura di «Arsi gran tempo e del mio foco indegno» di Torquato Tasso, in «Italique», XV (2012), pp. 47-72.

TOMASI 2015:

Franco TOMASI, Osservazioni sul libro di poesia nel secondo Cinquecento (156o1602), in Canzonieri in transito: lasciti petrarcheschi e nuovi archetipi letterari 
tra Cinque e Seicento, a c. di Alessando Metlica e Franco Tomasi, Milano-Udine, Mimesis, 2015, pp. 11-36.

TOMASI 2019:

Franco TOMASI, Teoria delle passioni ed esegesi lirica: le lezioni sulla gelosia di Benedetto Varchi, in «Annali della Scuola superiore di Pisa», V Serie, 11 (2019), 2, pp. 493-510.

TONELLI 2015:

Natascia TONELLI, Fisiologia della passione. Poesia d'amore e medicina da Cavalcanti a Boccaccio, Firenze, Sismel, 2015.

VASSALLI 1989:

Antonio VASSALLI, Falsirena in musica: un'altra redazione del soliloquio d'amore, in Lectura Marini, a c. di Francesco Guardiani, Toronto, Dovehouse editions Inc., 1989, pp. 201-11.

VILLANOVA 1585:

Arnaldo DA Villanova, De Amore heroico, in Opera omnia, ex officina Pernea per Conradum Waldkirch, Basilea, 1585, pp. 1523-30.

VITO 1585:

Niccolò VITO, Lezione della gelosia letta nella Academia fiorentina, Orvieto, per Baldo Salviani, 1585 .

WERNER 1993:

Gundersheimer WERNER, "The Green-Eyed Monster": Renaissance Conceptions of Jealousy, in "Proceedings of the American Philosophical Society», 137 (1993), 3, pp. 321-31.

ZUCCOLO 1615:

Ludovico ZucColo, Il Cataneo overo della gelosia, in Dialoghi di Lodouico Zuccolo, Perugia, appresso Annibale Aluigi \& fratelli, 1615, pp. 149-56.

Siti web

REPIM:

$<$ http://repim.muspe.unibo.it/risultati.aspx >, Repertorio della Poesia Italiana in Musica. [09 settembre 2021] 